\title{
ANÁLISE COMPARATIVA DA VIABILIDADE ECONÔMICA DE PLANTIOS DE Pinus taeda E Eucalyptus dunnii NA REGIÃO CENTRO- SUL DO PARANÁ
}

\author{
Vinicius Vitale$^{1}$, Gabriel de Magalhães Miranda ${ }^{2}$ \\ ${ }^{1}$ Eng. Florestal, Mestrando em Eng. Florestal, UNICENTRO, Irati, PR, Brasil - viniflorestal@ hotmail.com \\ ${ }^{2}$ Eng. Florestal, Dr., Depto. de Engenharia Florestal, UNICENTRO, Irati, PR, Brasil - gmiranda@ irati.unicentro.br \\ Recebido para publicação: 21/10/2008 - Aceito para publicação: 22/02/2010
}

\begin{abstract}
Resumo
O presente trabalho teve por objetivo fazer uma análise comparativa de viabilidade econômica de projetos florestais com Pinus taeda e Eucalyptus dunnii localizados no município de Prudentópolis, PR. Na análise econômica foram utilizados os critérios do Valor Presente Líquido (VPL), Razão Benefício Custo (B/C), Taxa Interna de Retorno (TIR) e Valor Periódico Equivalente (VPE). A taxa de juros usada foi de 6,75\% ao ano. Para o projeto de Pinus taeda os dados de custos e receitas corrigidos totalizaram $\mathrm{R} \$ 4.344,71 / \mathrm{ha}$ e $\mathrm{R} \$ 36.143,99 / \mathrm{ha}$, respectivamente. Os valores calculados dos critérios de avaliação econômica foram um VPL de R $\$ 31.799,28$ /ha; Razão Beneficio/Custo de 8,32, TIR de $27.23 \%$ e VPE de R $3.200,87 /$ ha/ano. Para o povoamento de Eucalyptus dunnii, a correção dos valores para o momento zero totalizaram $\mathrm{R} \$ 5.767,75 / \mathrm{ha}$, de custos e $\mathrm{R} \$ 43.842,46 / \mathrm{ha}$ de receitas. Os valores calculados dos critérios de avaliação econômica foram um VPL de R $\$ 38.074,71 /$ ha; Razão Beneficio/Custo de 7,60; TIR de 35,83\% e um VPE de R \$3.832,55/ha/ano. Para os dois projetos foi realizada análise de sensibilidade do VPL. Os resultados mostraram que ambos os projetos são economicamente viáveis.
\end{abstract}

Palavras-chave: Análise comparativa; viabilidade econômica; custos; receitas.

\begin{abstract}
Comparative analysis of the economic viability of Pinus taeda and Eucalyptus dunnii plantations in the south-center region of Parana State, Brazil. The objective of this research was to carry out a comparative analysis of economic viability of afforestation projects with Pinus taeda and Eucalyptus dunnii located in Prudentópolis County, State of Paraná, Brazil. In the economic analysis the criteria of Net Present Value (NPV), Benefit Cost rate (B/C), Internal Return Rate (IRR) and Periodic Equivalent Value (PEV) were used. The interest rate used was of $6.75 \%$ per the year. For the Pinus taeda plantations the data of rectified costs and income totalized R \$ 4.344,71/ha and R\$ $36.143,99 /$ ha, respectively. The calculated values of the of economic evaluation criteria presented a NPV of R $\$ 31,799.28 /$ ha, a Benefit Cost rate of 8,32 , an IRR of $27.23 \%$ and a PEV of R\$ $3.200,87 /$ ha. For the Eucalyptus dunnii plantations the rectified values totalized R $\$ 5.767,75$ and $\mathrm{R} \$ 43.842,46 /$ ha for the costs and income, respectively. The calculated values of the economic evaluation criteria showed a NPV of R $\$ 38.074,71 /$ ha, a Benefit Cost rate of 7.60, an IRR of 35,83\%, and a PEV of R $\$ 3.832,55 / \mathrm{ha}$. A sensitivity analysis of the NPV for the two plantations was carried out. The results showed that both afforestation projects are economically viable.

Keywords: Comparative analysis; economic viability; costs; income.
\end{abstract}

\section{INTRODUÇÃO}

A diversificação da produção é uma estratégia interessante para os pequenos produtores, sob dois aspectos: a possibilidade de receitas mais distribuídas ao longo do ano e a não dependência de uma ou poucas atividades, que, em caso de ocorrência de algum sinistro, problemas de mercado ou outro qualquer, podem comprometer o desempenho da propriedade. Nesse contexto, inserem-se as culturas florestais, que podem ser adotadas na forma de monoculturas ou em consórcios com culturas agrícolas ou animais. 
As espécies dos gêneros Pinus e Eucalyptus, quando comparadas a outras espécies, apresentam grande versatilidade para crescer e produzir madeira em variados tipos de ambiente, bem como permitem uma multiplicidade de usos da sua madeira. Assim sendo, essas espécies possibilitam a geração dessa matéria-prima madeireira, em todo o território nacional, em substituição àquela obtida com espécies nativas.

O desenvolvimento da tecnologia de utilização da madeira e a ampliação das alternativas de uso tornaram algumas espécies desses gêneros cada vez mais demandadas no setor florestal. Em decorrência disso, vem aumentando o número de produtores, especialmente pequenos e médios proprietários rurais, interessados no plantio e manejo de povoamentos florestais com tais espécies.

Seguindo a tendência mundial, várias indústrias instalaram-se no sul do Brasil, objetivando a produção e beneficiamento de madeira de reflorestamento. Essas indústrias nem sempre têm autossuficiência no abastecimento da matéria-prima de origem florestal, necessitando, assim, da madeira produzida pelos pequenos produtores rurais da região.

Nesse contexto, a aplicação dos critérios de análise econômica na área florestal é fundamental para se decidir qual o melhor projeto e/ou alternativa de manejo a serem adotados. Mais especificamente, a determinação da idade econômica de corte, o espaçamento, a adubação, a época e intensidade de tratamentos silviculturais e a espécie, entre outras decisões, podem ser adotados de forma mais segura quando feitas as simulações baseadas em critérios técnico-econômicos (LOPES, 1990).

Muitos trabalhos têm sido feitos sobre análise econômica de projetos florestais, a maioria deles utilizando os principais indicadores da viabilidade econômica: Valor Presente Líquido (VPL), Taxa Interna de Retorno (TIR), Razão Benefício/Custo (B/C), Valor Anual Equivalente (VAE) e Custo Médio de Produção (CMP) (NAUTIYAL, 1988; REZENDE; OLIVEIRA, 2001). Todos esses critérios levam em conta a variação do capital no tempo, mas cada um aponta diferentes aspectos relacionados aos projetos.

Este trabalho teve por objetivo fazer uma análise comparativa da viabilidade econômica de dois projetos de Pinus taeda e Eucalyptus dunnii no município de Prudentópolis, PR.

\section{REVISÃO BIBLIOGRÁFICA}

\section{Economia florestal}

Silva et al. (2005) definem economia florestal como o ramo da ciência que trata da utilização racional de recursos com vistas à produção, à distribuição e ao consumo de bens e serviços florestais. Portanto pode-se dizer, ainda, que a economia florestal procura resolver problemas do setor florestal, como compra, venda, taxação, manejo de florestas e seus subprodutos.

Já o manejo sustentável das florestas envolve as atividades relacionadas a implantação, crescimento, manutenção, colheita e comercialização da produção. Portanto, para se obter sucesso nesse manejo, é necessário o conhecimento das forças econômicas, ecológicas, sociais e políticas que determinam o uso racional dos recursos florestais (SILVA et al., 2005).

Silva et al. (2005) complementam seu estudo argumentando que a avaliação dos projetos florestais deve-se basear em seu fluxo de caixa, que consiste nos custos e nas receitas distribuídos ao longo da vida útil do empreendimento. Se as receitas forem maiores que os custos, então tem-se um projeto com viabilidade econômica.

Para Rezende; Oliveira (2001), o fluxo de caixa é considerado uma sequência de números reais $\mathrm{X}_{\mathrm{j}}$, que representa a receita líquida obtida em cada período $\mathrm{j}$, sendo que $\mathrm{j}$ varia de 0 a n períodos.

Silva et al. (2005) citam que existem vários métodos de avaliação de um projeto florestal. Cada um toma como base determinadas premissas, e não há consenso de qual método é mais indicado. Para os autores, no grupo dos critérios que consideram a variação do capital no tempo, são relacionados o Valor Presente Líquido (VPL), a Razão Benefício/Custo (B/C), a Taxa Interna de Retorno (TIR) e o Valor Periódico Equivalente (VPE). Pode ser usado, ainda, o Valor Esperado da Terra (VET) ou Valor Esperado do Solo (VES) como indicador da viabilidade de um projeto florestal.

\section{Critérios para a avaliação econômica}

Rezende; Oliveira (2001) mencionam que todo projeto, antes de ser implementado, deve submeter-se a um teste de viabilidade econômica, que consiste em verificar se as receitas inerentes ao projeto superam os custos necessários. Saliente-se que tanto os custos como as receitas são valores diretos, observados do ponto de vista privado. 
A análise econômica de um investimento envolve o uso de técnicas e critérios de avaliação que comparam os custos e as receitas inerentes ao projeto, visando decidir se ele deve ou não ser implementado (REZENDE; OLIVEIRA, 2001).

\section{Valor Presente Líquido (VPL)}

O VPL, de acordo com Silva et al. (2005), é a diferença entre o valor presente das receitas e o valor presente dos custos. Para a obtenção do seu valor, é adotada a seguinte expressão matemática:

$$
V P L=\sum_{j=o}^{n} R_{j}(1+i)^{-j}-\sum_{j=o}^{n} C_{j}(1+i)^{-j}
$$

Em que: $R_{j}=$ receita no período $j$;

$C_{j}=$ custo no período $j$

$i=$ taxa de juros;

$j=$ período de ocorrência da receita ou do custo $(0 \ldots n)$;

$n$ = número máximo de períodos de duração do projeto.

A característica essencial do método do VPL é o desconto para o presente de todos os valores esperados como resultado de uma decisão de investimento. Isso serve para satisfazer o requisito básico segundo o qual as opções devem ser comparadas somente se as consequências monetárias forem medidas em um ponto comum no tempo (REZENDE; OLIVEIRA, 2001). Esses mesmos autores comentam, também, que, para o uso do VPL, faz-se necessária a definição de uma taxa de desconto. Porém a seleção de um valor apropriado para a taxa de desconto é um dos problemas do uso do método do VPL. Esse método é muito sensível a mudanças na taxa de desconto, especialmente no caso de projetos de longo prazo. Pequenas taxas de desconto podem alterar a classificação dos projetos e as conclusões referentes à sua lucratividade. Faro (1979), por sua vez, menciona que projetos considerados antieconômicos, analisados numa determinada taxa de juros, podem se tornar viáveis quando avaliados numa taxa menor. Além disso, a mudança na taxa de juros pode acarretar uma variação na ordenação de alternativas.

O VPL é um dos melhores métodos, pois apresenta menos falhas e, na maioria das vezes, conduz ao resultado correto. Porém o método não considera o horizonte do projeto, e, por isso, se os projetos analisados possuírem diferentes durações ou tempos de maturação, há necessidade de correção dos horizontes (REZENDE; OLIVEIRA, 2001).

\section{Taxa Interna de Retorno (TIR)}

A TIR, como relata Silva et al. (2005), é a taxa de desconto que iguala o valor presente das receitas ao valor presente dos custos, ou seja, iguala o VPL a zero. Além disso, a TIR pode, também, ser entendida como a taxa percentual do retorno do capital investido. Sua fórmula é dada por:

$$
\sum_{j=o}^{n} R_{j}(1+T I R)^{-j}=\sum_{j=o}^{n} C_{j}(1+T I R)^{-j}
$$

Em termos das vantagens da utilização da TIR, tem-se que, com a adoção dessa técnica, não é preciso estimar a taxa de juros e é um bom critério para comparar alternativas de investimentos.

\section{Razão Benefício/Custo (B/C)}

Esse critério estabelece a relação entre o valor atual das receitas e o valor atual dos custos. O cálculo dessa razão é feito por meio da seguinte fórmula:

$$
B / C=\frac{\sum_{j=o}^{n} R_{j}(1+i)^{-j}}{\sum_{j=o}^{n} C_{j}(1+i)^{-j}}
$$

De acordo com Rezende; Oliveira (2001), pode-se dizer que, de forma geral, quando a razão $\mathrm{B} / \mathrm{C}>1$, o VPL é maior que 0 e a TIR é maior que a taxa do projeto. Uma das observações a serem feitas 
sobre esse método é que, assim como os métodos do VPL e da TIR, ele não leva em conta a duração dos projetos, pois apenas considera a relação entre custo e beneficio.

\section{Valor Periódico Equivalente (VPE)}

O Valor Periódico Equivalente (VPE) é a parcela periódica e constante necessária ao pagamento de uma quantia igual ao VPL da opção de investimento em análise, ao longo de sua vida útil. A relevância da aplicação do método do VPE encontra-se na seleção de projetos que apresentam durações ou vidas úteis diferentes, visto que os valores equivalentes obtidos por período corrigem, implicitamente, as diferenças de horizonte (FERREIRA, 2001).

Silva et al (2005) salientam que esse critério transforma o valor atual do projeto ou seu VPL em fluxo de receitas ou custos periódicos e contínuos, equivalente ao valor atual durante a vida útil do projeto.

É apresentado pela seguinte formula:

$$
V P E=\frac{V P L \times(1+i)^{t}}{1-\frac{1}{(1+i)^{n t}}}
$$

Para se determinar o Valor Periódico Equivalente, é necessário, primeiramente, obter o VPL de cada projeto e sua duração.

Esse critério permite comparar projetos de durações diferentes, sendo essa uma de suas grandes vantagens.

\section{MATERIAL E MÉTODOS}

\section{Caracterização da área de estudo}

Os dados para a realização deste estudo são oriundos de plantios de $P$. taeda e E. dunnii no município de Prudentópolis, região centro-sul do estado do Paraná, localizado geograficamente a $25^{\circ} 12^{\prime} 46^{\prime \prime} \mathrm{S}$ e $50^{\circ} 58^{\prime} 40^{\prime \prime} \mathrm{O}$.

Segundo a classificação climática de Köppen, a região apresenta clima do tipo $\mathrm{Cfb}$, com geadas frequentes no inverno. A temperatura média máxima é de $24,2{ }^{\circ} \mathrm{C}$ e a média mínima é de $11,0{ }^{\circ} \mathrm{C}$. A precipitação média mensal é de $193,97 \mathrm{~mm}$, e a umidade relativa do ar média mensal é de $79,58 \%$. A altitude é de $840 \mathrm{~m}$.

\section{Dados de custos e receitas}

Foram considerados os custos por hectare de implantação (aquisição de mudas, preparo do solo, combate inicial a formigas, plantio e controle de plantas invasoras, entre outros), custos de manutenção (por exemplo, roçada, coroamento, manutenção de aceiros e combate permanente a formigas), custos com podas e alguns custos eventuais. As receitas foram as provenientes da venda da madeira de desbastes e do corte final, tomando-se como base a venda da madeira em pé, sem contemplar, portanto, os custos de colheita.

\section{Organização dos dados}

Para efeito da análise econômica, utilizou-se a taxa de juros de 6,75\% ao ano, adotada no Programa de Plantio Comercial e Recuperação de Florestas (PROPFLORA), pelo Banco Nacional de Desenvolvimento Econômico e Social (BNDES), vigente até 30/06/2009 (BNDES, 2008).

Os dados de custos e receitas foram considerados como valores correntes (valores reais no momento de ocorrência dos gastos) e valores corrigidos (valores corrigidos para o momento zero do horizonte de planejamento, de acordo com a taxa de juros adotada).

Os custos de implantação foram considerados todos ocorrendo no período zero, e os custos de manutenção e de poda ocorrendo do ano zero até o fim da rotação, aos 17 anos.

Os dados foram organizados em fichas de campo, com a descrição da atividade realizada, o ano de ocorrência, a quantidade executada e os preços unitários e totais pagos por ocasião da realização. 
Admitindo-se que as podas não foram realizadas em toda a área em todos os períodos e que os valores observados dos custos de manutenção e podas foram irregulares ao longo do horizonte de planejamento, para efeito de simplificação do fluxo de caixa, os valores de custos observados nos respectivos períodos de ocorrência dessas atividades foram descapitalizados para o período zero do horizonte de planejamento e depois foram transformados em valores periódicos constantes, por meio de uma série de pagamentos temporária, constante, imediata postecipada, fazendo com que o valor pudesse ser citado uma única vez e relacionado a todos os períodos.

\section{Processamento dos dados e critérios econômicos de decisão}

Os dados foram processados utilizando-se planilhas do Microsoft Office Excel 2007, e os povoamentos foram avaliados de acordo com os seguintes critérios de avaliação econômica, com as respectivas considerações de Silva et al. (2005):

Valor Presente Líquido (VPL)

O projeto que apresenta VPL maior que zero é considerado economicamente viável, e na comparação de projetos é considerado melhor aquele que apresentar o maior VPL.

\section{Taxa Interna de Retorno (TIR)}

A avaliação com base na TIR considera viável o projeto em que ela for maior que a taxa de juros utilizada nos cálculos, e na comparação de projetos será considerado melhor aquele que apresentar a maior TIR. Uma outra consideração que pode ser feita em relação à TIR, no que diz respeito à tomada de decisão, é a comparação com uma taxa mínima de atratividade, que pode ser a taxa de uma aplicação financeira alternativa, ou a taxa de retorno de um projeto alternativo, ou, ainda, a taxa mínima de retorno estipulada pelo empreendedor para que o projeto seja atraente.

\section{Razão Benefício/Custo (B/C)}

O projeto é economicamente viável se apresentar razão B/C $>1$. Na comparação de projetos, aquele que apresentar a maior relação será considerado o melhor. $\mathrm{O}$ valor calculado $\mathrm{B} / \mathrm{C}$ representa a rentabilidade proporcionada pelo investimento, ou seja, representa o número de unidades monetárias retornadas para cada unidade monetária investida, já corrigida pela taxa de juros.

\section{Valor Periódico Equivalente (VPE)}

Partindo-se do pressuposto que o VPL representa o valor atual do projeto, o VPE transforma esse valor único em um fluxo de valores periódicos (custos ou receitas) constantes, distribuídos de forma homogênea ao longo do horizonte de planejamento do projeto.

O projeto será considerado economicamente viável se apresentar VPE positivo, e na comparação de projetos será considerado melhor aquele que apresentar maior VPE.

\section{Análise de sensibilidade}

Rezende; Oliveira (2001) mostram que a relação entre a taxa de juros e o VPL é inversa, ou seja, quanto maior a taxa de juros, menor o VPL e vice-versa. Com base nessa constatação, foi realizada uma análise da sensibilidade do VPL em função da variação da taxa de juros.

A análise de sensibilidade foi feita com base no VPL, variando as taxas de juros de $1 \%$ até $44 \%$ ao ano, com variações pontuais de $1 \%$ ao ano. Esse tipo de estudo mostra o quanto os projetos são afetados pela variação da taxa de juros, possibilitando até identificar graficamente uma aproximação da taxa de juros que marca a interface entre os intervalos de taxas de juros que determinam a viabilidade ou inviabilidade de projetos, ou seja, uma aproximação da TIR.

Para efeito de ilustração, foram tomadas como exemplo as taxas de 1, 10, 20, 30 e $40 \%$ ao ano, e com os resultados foram gerados dois gráficos, um para cada espécie em estudo. 


\section{RESULTADOS E DISCUSSÃO}

\section{Análise econômica}

A análise econômica dos projetos foi realizada por espécie, e em seguida foi feita a comparação entre as espécies. Os dados de custos e receitas dos povoamentos foram corrigidos para o momento zero, como mostra a tabela 1 .

Tabela 1. Valores correntes e corrigidos de custos e receitas dos projetos de P. taeda e E. dunnii. Table 1. Current and corrected values of costs and revenues of the projects of P. taeda and E. dunnii.

\begin{tabular}{|c|c|c|c|c|c|c|c|c|c|}
\hline \multirow{3}{*}{ Período } & \multirow{3}{*}{ Atividade } & \multicolumn{4}{|c|}{ Pinus taeda } & \multicolumn{4}{|c|}{ Eucalyptus dunnii } \\
\hline & & \multicolumn{2}{|c|}{ Custos (R\$/ha) } & \multicolumn{2}{|c|}{ Receitas (R\$/ha) } & \multicolumn{2}{|c|}{ Custos (R\$/ha) } & \multicolumn{2}{|c|}{ Receitas (R\$/ha) } \\
\hline & & Corrente* & Atual** & Corrente* & Atual** & Corrente* & Atual** & Corrente* $^{*}$ & Atual** \\
\hline 0 & Implantação & $1.504,00$ & $1.504,00$ & & & $2.422,20$ & $2.422,20$ & & \\
\hline 1 a 17 & Manutenção & 189,06 & $1.878,19$ & & & 159,89 & $1.588,49$ & & \\
\hline 1 a 17 & Podas & 57,20 & 568,00 & & & 124,08 & $1.232,72$ & & \\
\hline 1 a 17 & Gastos eventuais & 39,71 & 394,52 & & & 52,78 & 524,34 & & \\
\hline 5 & Desbaste & - & - & - & - & & & $10.000,00$ & $7.213,74$ \\
\hline 11 & Desbaste & & & $24.716,00$ & $12.048,46$ & & & $40.000,00$ & $19.499,04$ \\
\hline 17 & Corte raso & & & $73.146,00$ & $24.095,53$ & & & $52.000,00$ & $17.129,68$ \\
\hline Total & & & $4.344,71$ & & $36.143,99$ & & $5.767,75$ & & $43.842,46$ \\
\hline
\end{tabular}

Fonte: Produtores rurais.

*Valor observado no momento de ocorrência da atividade; **Valor corrigido pela taxa de juros para o momento zero do horizonte de planejamento.

A análise realizada indicou que o projeto de $P$. taeda obteve valores presentes para os custos de $\mathrm{R} \$ 4.344,71 /$ ha e de $\mathrm{R} \$ 36.143,49 /$ ha para as receitas, resultando em um VPL de $\mathrm{R} \$ 31.799,28 / \mathrm{ha}$. Esses valores demonstram que o projeto de $P$. taeda, nas condições avaliadas, é uma atividade economicamente viável.

Para o projeto de E. dunnii, os valores observados foram de $\mathrm{R} \$ 5.767,75 /$ ha e $\mathrm{R} \$ 43.842,46 /$ ha para custos e receitas, respectivamente. Com isso, obteve-se um VPL de R \$ 38.074,71.

Esses resultados permitem afirmar que, pelo critério do VPL, ambos os projetos são economicamente viáveis, e que, quando comparados, o projeto de $E$. dunnii mostrou-se economicamente mais vantajoso que o projeto de $P$. taeda, por apresentar maior VPL.

Pelo método da Razão Benefício/Custo, os resultados indicaram uma rentabilidade de 8,32 e 7,60 vezes o valor investido, para $P$. taeda e E. dunnii, respectivamente.

Os valores de TIR observados indicaram que, nas condições analisadas, os projetos proporcionaram retornos de $27,23 \%$ e de $35,83 \%$ ao ano, para $P$. taeda e $E$. dunni, respectivamente. A TIR sendo maior que a taxa do projeto indica a viabilidade econômica do empreendimento. Portanto os valores encontrados confirmam os resultados dos outros critérios de avaliação utilizados, no que diz respeito à viabilidade dos projetos. Outra consideração que pode ser feita em relação à TIR é a comparação dela com uma taxa mínima de atratividade, que pode ser a taxa de uma aplicação financeira alternativa, ou a taxa de retorno de um projeto alternativo, ou, ainda, a taxa mínima de retorno estipulada pelo empreendedor para que o projeto seja atraente.

O VPE apresentou valores de $\mathrm{R} \$ 3.200,87 / \mathrm{ha} /$ ano e $\mathrm{R} \$ 3.832,55 / \mathrm{ha} /$ ano para $P$. taeda e E. dunnii, respectivamente.

Os indicadores de viabilidade econômica VPL, B/C, TIR e VPE verificados para as duas espécies são mostrados na tabela 2.

Tabela 2. Indicadores de viabilidade econômica calculados para os projetos das duas espécies avaliadas. Table 2. Calculated pointers of economic viability for the projects of the two evaluated species.

\begin{tabular}{lccccccc}
\hline \multicolumn{4}{c}{ Pinus taeda } & \multicolumn{4}{c}{ Eucalyptus dunnii } \\
\hline VPL & B/C & TIR & VPE & VPL & B/C & TIR & VPE \\
\hline R $\$ 31.799,28 / \mathrm{ha}$ & 8,32 & $27,23 \%$ & $\mathrm{R} \$ 3.200,87 \mathrm{ha} / \mathrm{ano}$ & $\mathrm{R} \$ 38.074,71 / \mathrm{ha}$ & 7,6 & $35,83 \%$ & $\mathrm{R} \$ 3.832,55 \mathrm{ha} / \mathrm{ano}$ \\
\hline
\end{tabular}

VPL: Valor Presente Líquido; B/C: Razão Benefício/Custo; TIR: Taxa Interna de Retorno; VPE: Valor Periódico Equivalente. 
A figura 1 mostra a distribuição percentual dos valores presentes dos custos dos dois projetos, por atividade.

Considerando-se que a variação da taxa de juros altera a viabilidade dos projetos, a análise de sensibilidade é importante, pois possibilita a visualização do comportamento do parâmetro indicador de viabilidade em relação à variação dos juros.

O ponto em que a curva do VPL intercepta o eixo das abscissas (eixo da taxa de juros) indica a taxa na qual o projeto tem o VPL igual a zero, ou seja, a taxa de juros que representa a TIR. A análise de sensibilidade dos projetos das duas espécies em relação ao VPL é mostrada na figura 2.
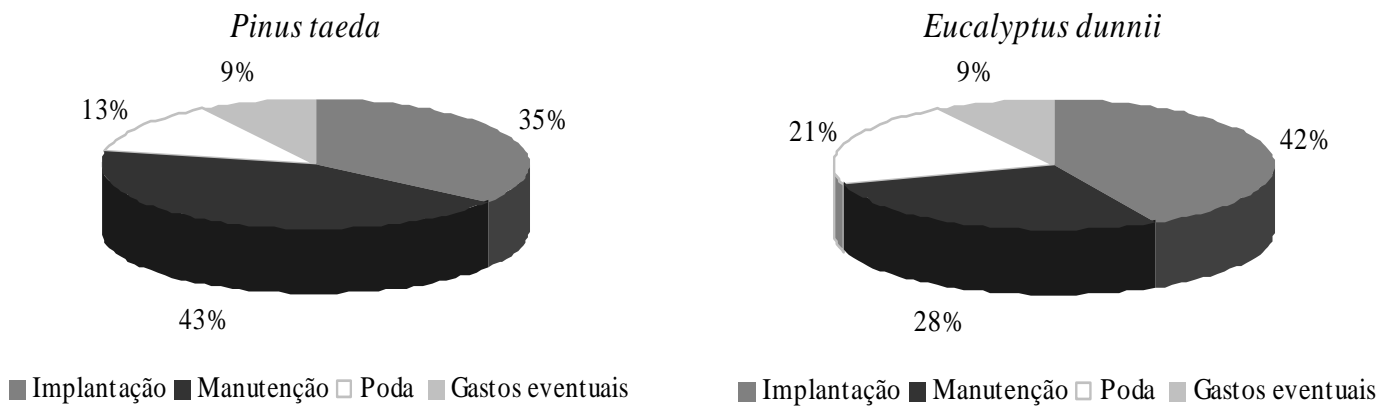

Figura 1. Distribuição percentual dos custos corrigidos dos projetos de Pinus taeda e Eucalyptus dunnii para o momento zero do horizonte de planejamento.

Figure 1. Percentual apportionment of the corrected costs of the projects of Pinus taeda and Eucalyptus dunnii for nought moment from the projection horizon.

\section{Análise de sensibilidade}
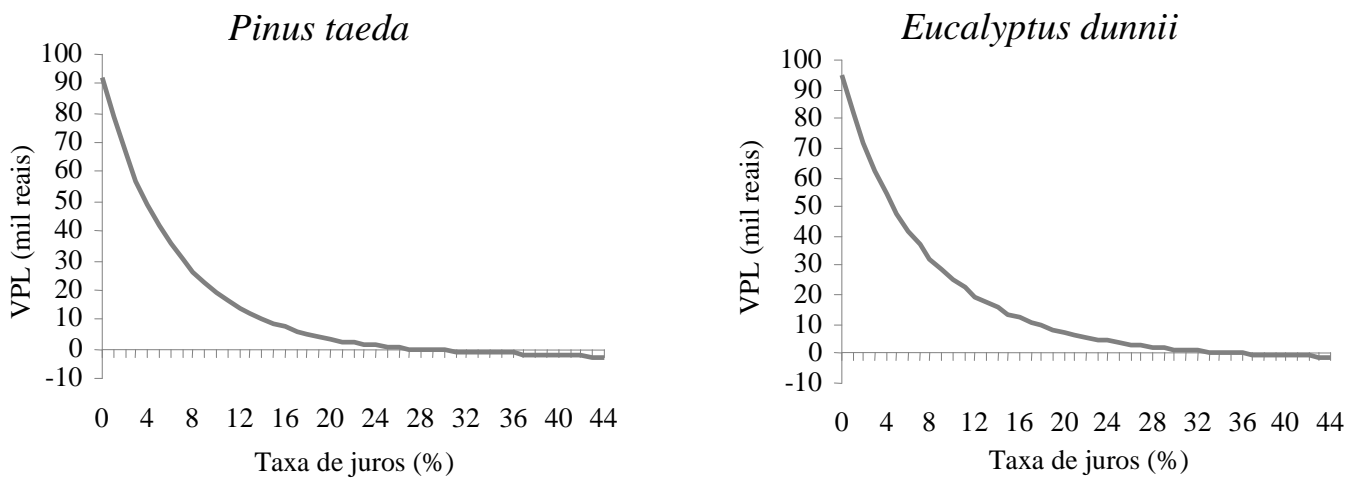

Figura 2. Tendência do VPL dos projetos de Pinus taeda e Eucalyptus dunnii em resposta à variação da taxa de juros.

Figure 2. Bias from the NPV from the projects of Pinus taeda and Eucalyptus dunnii in reply to the variation of the interest rate.

O povoamento de $P$. taeda, submetido às taxas de juros propostas de 1, 10, 20, 30 e $40 \%$ ao ano, apresentou VPL's de R \$ 78.532,22/ha, R \$ 19.169,16/ha, R \$3.365,83/ha, R \$-658,31/ha e R \$-2.108,52/ha, respectivamente. O projeto de $E$. dunnii, por sua vez, apresentou VPL's de R\$ 82.309,86/ha, $\mathrm{R} \$ 25.195,93 / \mathrm{ha}, \mathrm{R} \$ 7.276,72 / \mathrm{ha}, \mathrm{R} \$ 1.518,55 /$ ha e R $\$-702,82 /$ ha, respectivamente. 


\section{CONCLUSÕES}

As análises realizadas possibilitam chegar às seguintes conclusões:

- Apesar da característica de longo prazo, a atividade florestal é uma boa alternativa de diversificação da produção e da renda para pequenas propriedades, uma vez que as análises indicaram a viabilidade econômica dos projetos analisados.

- Quando analisados os povoamentos pelos métodos do VPL, TIR e VPE, o projeto com E. dunnii mostrou-se economicamente melhor, em comparação com projeto de $P$. taeda.

- A inversão na ordem de viabilidade dos projetos observada quando a avaliação foi feita pela Razão $\mathrm{B} / \mathrm{C}$ ocorreu pelo fato de o projeto de E. dunnii ter apresentado receitas e custos proporcionalmente maiores que o projeto de $P$. taeda, o que acabou resultando em uma relação menor.

- A TIR verificada para projeto de $E$. dunnii indica que ele continuaria economicamente viável mesmo no intervalo de taxas entre $27,23 \%$ a.a. e $35,83 \%$ a.a., que inviabilizariam economicamente o projeto de P. taeda.

- O melhor desempenho observado para o projeto de $E$. dunnii em relação ao de $P$. taeda pode ser explicado pelo fato de que, além de o primeiro ter apresentado receitas notadamente maiores, ele apresentou uma receita em um período de tempo menor, aos cinco anos, receita esta que acabou sofrendo uma menor influência da taxa de juros.

- A análise de sensibilidade comprovou a tendência de relação inversa existente entre o VPL e a taxa de juros, e que, para as condições estudadas, o projeto com $P$. taeda mostrou uma maior sensibilidade em relação às variações da taxa de juros, quando comparado com o projeto de $E$. dunnii.

\section{REFERÊNCIAS}

BNDES - BANCO NACIONAL DE DESENVOLVIMENTO ECONOMICO E SOCIAL (Brasil). Propflora. Disponível em:<http://www.bndes.gov.br/programas/agropecuarios/propflora.asp>. Acesso em: 24/3/2008.

FARO, C. de. Elementos de engenharia econômica. 3.ed. São Paulo: Atlas, 1979. 328 p.

FERREIRA, T. C. Análise econômica de plantios de eucalipto para a produção de celulose. Dissertação (Mestrado em Engenharia Florestal) - Universidade Federal de Lavras, Lavras, 2001.

LOPES, H. V. S. Análise econômica dos fatores que afetam a rotação de povoamentos de eucaliptos. 1990. 188 f. Dissertação (Mestrado em Ciência Florestal) - Universidade Federal de Viçosa, Viçosa, MG, 1990.

NAUTIYAL, J. C. Forest economics: principles and applications. Toronto: Canadian Scholars' Press Inc., 1988. $581 \mathrm{p}$.

REZENDE, J. L. P.; OLIVEIRA, A. D. Análise Econômica e Social de Projetos Florestais. Viçosa, MG: UFV, 2001. 389 p.

SILVA, M. L.; JACOVINE, L. A. G.; VALVERDE, S. R. Economia florestal. 2 ed. Viçosa, MG: UFV, 2005. $178 \mathrm{p}$. 